\title{
Zur Altersstaffelung von Säugetierresten und der Frage paläolithischer Jagdbeute
}

\author{
Von Elisabeth Schmid, Freiburg i. Br.
}

$\mathrm{Z}$ u s a m m e $\mathrm{f}$ a s sung. Dem durch H. BäCHLFR statistisch festgestellten Überwiegen von Jungtieren in einigen Höhlenbären-Höhlen als Beweis für paläolithische Jagd wird die Beobachtung von D. BuRCKHARDT gegenïbergestellt, daß in der Umgebung des Schweizerischen Nationalparkes bei dem Hirschsterben während schneereicher Winter die Jungtiere weitaus den größten Anteil ausmachen. Als Todesursache ist hier Verhungern festgestellt. Da der Anteil an Jungtieren in den Höhlenbären-Höhlen umso größer ist, je höher die Höhle liegt, da zudem die Knochenlager der Höhlenbären vom Ende des Riß/Würm-Interglazials an vor allem in der Vorrückungszeit der Würm-Vereisung sedimentiert worden sind, kann das Vorherrschen von Jungtieren durchaus in der Mangelernährung während schlechter Sommer und in den langen Wintern beruhen. Es wird die Forderung nach stärkerer Berücksichtigung der vielfältigen Erkenntnisse der modernen Säugetier-Zoologie bei der Deutung von Knochenlagern erhoben.

Ré s u mé. De la prédominance des oursons dans quelques "grottes à ours", H. BäCHLER a conclu à la certitude de la chasse paléolithique. L'auteur oppose à cette preuve les observations de D. Burckhardt dans la région du Parc National Suisse où, durant des hivers à fortes chûtes de neige, de nombreux cerfs ont péri, la proportion des faons étant la plus forte; l'analyse vétérinaire a montré que ces animaux sont morts de faim. Dans les cavernes, la proportion des oursons augmente avec l'altitude absolue du site; la sédimentation des ossements s'est effectuée à la fin de l'interglaciaire Riß/Würm et au crû de Würm. On peut conclure que la prédominance des oursons a pour cause le manque de nourriture résultant de mauvais étés et d'hivers très longs. L'auteur demande que, pour l'interprétation des ossements fossiles, on tienne davantage compte des connaissances de la zoologie moderne des mammifères.

I.

An allen Fundstellen pleistozäner Säugetierknochen muß die Frage geprüft werden, ob diese Knochen auf natürliche Weise oder mir Zutun des Menschen abgelagert worden sind. Wenn das Sediment Feuerstellen und zahlreiche Geräte enthält, wenn die Knochen Hack- oder Schnittspuren tragen oder häufig angebrannt sind, dann ist die Ansammlung der Säugetierknochen eindeutig auf die Jagdtätigkeit des Menschen zurückzuführen. Aber selbst wenn am Fundplatz Kulturhinterlassenschaften des Menschen, vor allem Geräte, auftreten, ist damit nicht immer erwiesen, daß alle oder auch nur einige der Tierknochen von der Jagdbeute des Menschen stammen, denn sowohl an Fluß- und Seeufern wie auch in Höhlen können Mensch und 'Tier abwechselnd aufgetreten sein, so daß die Tierreste natürliche Totengemeinschaften oder zumindest Einbettungsgemeinschaften darstellen. Jedoch ist es hierbei auch möglich, daß ein Teil der Knochen von Mahlzeitresten, der andere hingegen von natürlichen Abgängen stammt.

Liegt Jagdbeute vor, dann ist die Zusammensetzung der in den Knochen repräsentierten Fauna nicht ausschließlich durch das natürliche Vorkommen und Verenden der einzelnen Tierarten bestimmt. Vielmehr wirkt hier vor allem - innerhalb des von der Natur gegeben Rahmens - der Wille, die Sitte, die Jagdmethode des jagenden Menschen.

II.

Verschiedene Überlegungen und Analysen wurden schon vorgelegt, um ein eindeutiges Kennzeichen für die Entscheidung zu gewinnen, ob Jagd oder natürlicher Abgang vorliege.

Vor allem hat W. SoERges. (1922) die Altersstaffelung der einzelnen Tierarten hierfür ausgewertet, wobei er für die pleistozänen Elefanten von einer bekannten Jagdstation (Taubach) und dem Ort eines katastrophenartigen Untergangs einer Herde (Předmost) ausging. Dabei entstand die Regel, daß, wo die Altersstufen (sehr jung, jung, erwachsen, alt) etwa gleich verteilt sind, oder wo Jungtiere überwiegen, Jagdbeute des Menschen vor- 
liege. Wenn jedoch vor allem voll erwachsene Tiere den Knochenbestand ausmachen, dann sei der natürliche Tod der Tiere erwiesen. Diese Schlußfolgerung hat SoengEL auch auf andere Fundorte und Tiere übertragen, so daß er auf diese Weise z. B. in den Sanden von Mauer Jagdbeute des Heidelberger Archanthropinen (El. antiquus) gegenüber den natürlich verendeten (Rhin. merckii) ausscheiden konnte.

\section{III.}

Diese von Soengel vorgetragene Bearbeitungsweise fossiler Knochenlager zur Deutung ihrer Entstehungsweise ist unlängst durch H. B̈̈CHLER (1957) auf die Höhlenbärenreste der drei von seinem Vater E. BächlER (1940) ergrabenen Höhlen in den Schweizer Alpen übertragen worden. $\mathrm{H}$. Bächlen ging es darum, mit ihrer Hilfe die von seinem Vater wiederholt, zuletzt 1940 vorgetragene Ansicht, daß die in den Höhlen Drachenloch und Wildenmannlisloch gefundenen Knochen des Höhlenbären Beutereste des paläolithischen Jägers seien, und andererseits die Knochen vom Wildkirchli eine Mischung von Jagdbeute und natürlichen Abgängen darstelle, zu bekräftigen.

E. Bächlers These der Höhlenbärenjagd steht seit Jahrzehnten in zum Teil scharf geführtem wissenschaftlichem Streit. Wir wollen hier auf die zahlreichen Argumente der Gegner und Verteidiger, unter denen - zusammen genommen - vor allem die Namen E. Bächler, H. Bächler, H. Cramer, K. Ehrenberg, G. Freund, F. E. Koby, W. Soergel, H. G. Stehlin, L. F. Zotz genannt werden müssen, nicht näher eingehen.

Hingegen sei hier lediglich die oben genannte jüngste Untersuchung von H. Bächler (1957) kurz dargelegt und ihr eine andere Beobachtung angefügt.

\section{IV.}

In sorgfältiger Arbeit hat $\mathrm{H}$. B̈̈CHLER aus dem reichen Fundkomplex der drei unter seinem Vater ergrabenen Höhlen den oberen letzten großen Backenzahn $\left(\mathrm{M}^{2}\right)$ des Höhlenbären ausgelesen und das Sterbealter seines Trägers mit Hilfe der etwas erweiterten Marinelli'schen Altersstufen (1931, S. 390) statistisch festgehalten. Insgesamt hat H. BÄCHLER $992 \mathrm{M}^{2}$ untersucht, die sich etwa gleichmäßig auf die drei Höhlen verteilen, nämlich:

$\begin{array}{ll}\text { Wildkirchli } & 331 \text { Stück } \\ \text { Wildenmannlisloch } & 365 \text { Stück } \\ \text { Drachenloch } & 296 \text { Stück. }\end{array}$

H. B̈̈CHLER legt eine ausführliche Tabelle mit den absoluten Zahlen innerhalb der einzelnen Altersstufen, aufgeteilt in rechte und linke Zähne, vor und in einer zweiten Tabelle die entsprechenden Prozentwerte. Er faßt dann die acht Altersstufen in drei große Gruppen zusammen und erhält folgende Tabelle:

$\begin{array}{lccc} & \begin{array}{c}\text { Jungbären } \\ \text { (bis 4 Jahre) }\end{array} & \begin{array}{c}\text { Geschlechtsreife } \\ (5-15 j a ̈ h r i g)\end{array} & \text { Alte } \\ \text { Wildkirchli } & 41 \% & 24 \% & 35 \% \\ \text { Wildenmannlisloch } & 71 \% & 20 \% & 9 \% \\ \text { Drachenloch } & 80 \% & 15 \% & 5 \%\end{array}$

Das derart statistisch festgestellte starke Überwiegen der Jungbären im Wildenmannlisloch und Drachenloch legt $\mathrm{H}$. B̈̈CHLER als Beweis vor, daß alle Höhlenbärenknochen dieser beiden Höhlen Überreste der Beute des paläolithischen Jägers seien, wie andererseits der große Anteil erwachsener und vor allem alter Tiere den Misch-Charakter der Wildkirchlifunde dokumentiere, wo in der Oberen Höhle rein natïrliche Knochenlager, in den drei anderen Höhlenteilen (Wirtshaus-, Altar- und Kellerhöhle) hingegen Beutereste des Menschen mit vielleicht einzelnen, am Ort verendeten Bären angetroffen worden seien. 
$\mathrm{V}$.

Zur gleichen Zeit, als der Aufsatz von H. Bächler erschien, veröffentlichte D. BurckHARDT (1957) in der Zeitschrift des Schweizerischen Bundes für Naturschutz eine auf den Erfahrungen der letzten Jahre sich gründende Zusammenstellung „Über das Wintersterben der Hirsche in der Umgebung des Nationalparkes“.

Die Hirsche des Nationalparkes halten sich während des Winters nicht im Bereich des Parks auf, sondern wandern in die Umgebung ab. Hier sind während mehrerer Winter in Abständen von 1 oder 2 Jahren 20-200 tote Hirsche gemeldet worden. Die veterinärpathologische Untersuchung einer größeren Zahl dieser Hirsche ergab, daß keine Krankheit oder Degeneration vorlag, sondern daß „die Abgänge auf allgemeine Erschöpfung und Unterernährung zurückzuführen" seien. Mit diesem Ergebnis stimmt die Beobachtung überein, daß bis jetzt nur in schneereichen Wintern Rotwild in größerer Zahl verhungert ist. In Wintern mit geringer Schneedecke hingegen fanden die Tiere selbst in strengen Frostperioden genügend W'interäsung, so daß keine Hirsche umkamen.

Während des Wintersterbens $1952 / 53$ und 1954/55 sind 55 Schädel gesammelt und nach Geschlecht und Alter bestimmt worden. Der Vergleich mit dem Bestand lebender Tiere im Winter 1955/56 ergab folgende Liste (BurckнaRdт 1957, S. 2):

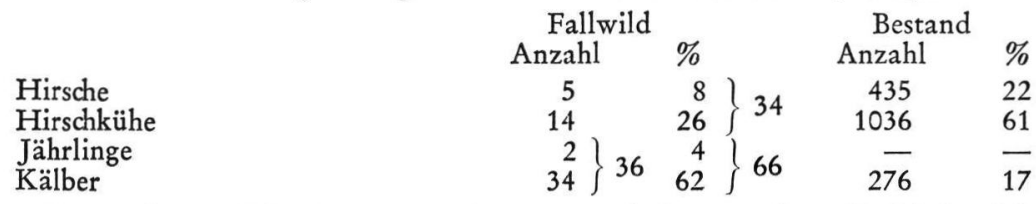

Einem Bestand von 17\% Jungtieren bei 83\% Alttieren stehen die Todesziffern $66 \%$ Jungtiere und $34 \%$ Alttiere gegenüber.

Wir können auf dieses Hirschproblem, das in dem Aufsatz ausführlich dargestellt ist, wobei auch auf die unvorstellbar großen Zahlen verhungerter Hirsche und zerstörter Wälder in Amerika hingewiesen wird, hier nicht näher eingehen. Uns interessiert im $\mathrm{Zu}-$ sammenhang mit dem pleistozänen Tiersterben vor allem das Zahlenverhältnis von Altund Jungtieren.

Hätten diese, ihrer Lebensweise nach zerstreut verendeten Tiere einen gemeinsamen Sterbeplatz, so würde ihre Altersstaffelung nach der SoERgEL'schen Regel einen Mahlzeitplatz von Jägern ergeben, wobei die Tatsache, daß im Hirschrudel die Jungen vorangehen, diese Deutung noch bestärken würde.

\section{VI.}

Wir sind uns voll bewußt, daß diese an den Hirschen gewonnene Erfahrung nicht direkt auf den Höhlenbären übertragen werden darf, ebensowenig wie Erkenntnisse beim fossilen Elefanten uneingeschränkt die Deutung von Höhlenbärenresten belegen können. Es handelt sich jeweils um andersartige Tiere mit anderen Lebensrhythmen und Verhaltensweisen. Aber Eines stellt sich unmittelbar heraus: Wenn viele Jungtiere sterben, dann $\mathrm{k}$ a $\mathrm{n} \mathrm{n}$ die Ursache darin liegen, daß sie verhungern.

Bei der Deutung der Höhlenbärenreste in Höhlen sollten weder die Besonderheiten der Lokalität noch Zeit und Dauer der Einbettung der Knochen außer Acht gelassen werden. So haben die drei von H. B̈̈CHLER untersuchten Höhlen folgende Höhenlage:

$\begin{array}{ll}\text { Wildkirchli } & 1486 \mathrm{~m} \text { ü. M. } \\ \text { Wildenmannlisloch } & 1628 \mathrm{~m} \text { ü. M. } \\ \text { Drachenloch } & 2445 \mathrm{~m} \text { ü. M. }\end{array}$

Die Höhlenbärenreste wurden im Drachenloch gegen Ende des Riß-Würm-Interglazials in das Sediment eingebettet (E. Schmid 1958). Die Höhle lag auch damals beträchtlich über der Waldgrenze. Daraus kann folgende Deutung abgelesen werden: Ungünstige Sommer 
schwächten vor allem die Jungtiere so stark, daß nur die kräftigsten die Zeit der langen Winterruhe in der Höhle überdauerten, die schwächeren aber verhungerten. Es hat demnach eine scharfe Auslese stattgefunden, so daß ein relativ geringer Prozentsatz erwachsen oder gar alt geworden ist.

Unter diesem Gesichtspunkt stellen wir aus den von H. BäCHLER gegebenen ausführlichen Werten (S. 142) folgende andere Kombination von Altersstufen zusammen, wobei vor allem bei den Jungbären daran gedacht wird, daß sie während der Wintermonate verendet sind:

\begin{tabular}{lcccc} 
Altersstufe & 1 & $2 \mathrm{a}+2 \mathrm{~b}$ & $2 \mathrm{c}+2 \mathrm{~d}+3$ & $4+5$ \\
\hline Lebensalter & $\begin{array}{c}3 / 4-1 \frac{1 / 4}{13 / 4-4} \\
\text { infantil }\end{array}$ & $\begin{array}{c}\text { juvenil } \\
\text { 1. Winter }\end{array}$ & $\begin{array}{c}\text { 2.-4. Winter } \\
\text { adult }\end{array}$ & $\begin{array}{c}\text { iber 20 } \\
\text { senil }\end{array}$ \\
\hline Wildkirchli & $24,5 \%$ & $16,3 \%$ & $42,9 \%$ & $16,3 \%$ \\
Wildenmannlisloch & $23,0 \%$ & $47,9 \%$ & $24,4 \%$ & $4,7 \%$ \\
Drachenloch & $17,2 \%$ & $63,5 \%$ & $18,6 \%$ & $0,7 \%$
\end{tabular}

Diese Tabelle führt zu dem Schluß, daß die Lebenserwartung des Höhlenbären umso geringer ist, je höher die Höhle liegt. Ferner: Den ersten Winter verbringt der Jungbär nach einem Sommer, während dem er teils gesäugt wird und teils unter der erfahrenen Betreuung seiner Mutter Kräfte sammeln konnte. In den folgenden Sommern aber gehen die jungen Bären zunehmend selbständig auf Nahrungssuche - und nur die findigsten und kräftigsten werden adult. Dividieren wir den jeweiligen Prozentwert der juvenilen Altersgruppen durch drei - die Anzahl der Winter, in denen sie verendet sind -, so ergibt sich durchschnittlich ein Abgang von

$\begin{array}{lr}\text { Wildkirchli } & 5,4 \% \\ \text { Wildenmannlisloch } & 16,0 \% \\ \text { Drachenloch } & 21,2 \% \text { pro Jahr. }\end{array}$

Der jährliche Abgang bei den Jungtieren in den späteren Jahren ist demnach nur im Drachenloch größer als im ersten Winter.

Daß eine derart starke Verringerung des Jungtierbestandes noch lange nicht zum Aussterben führen mußte, vermittelt eine einfache Überlegung (bei der wir stets an der unteren Grenze der möglichen Zahlen bleiben): Eine Bärin wirft 10 Jahre lang je 2 Junge, das sind 20 Junge in ihrem Leben. Zur Erhaltung des Bestandes ist es nur notwendig, daß 2 Tiere zur Geschlechtsreife und Fortpflanzung kommen. 18 Jungtiere können verenden. Nehmen wir an, daß drei Viertel von ihnen durch Unfälle oder als Beute von Raubtieren außerhalb der Höhle ihr Ende finden, - das wären etwa 14 Jungbären - dann können noch 4 Jungbären während der Wurffähigkeit der Bärin in der Höhle verenden, ohne daß der Bestand verringert wird. Würde die Bärin nach ihrer letzten Wurfperiode eingehen, dann bestünde die 'Totengemeinschaft in der Höhle im Verhältnis der Jungtiere zu dem Alttier wie $4: 1$ !

Es kann demnach sehr wohl eine in einer Höhle auch während langer Zeit gebildete Totengemeinschaft mit einem Vorwiegen von Jungtieren auf natürliche Ursachen zurückgeführt werden. Deshalb reichen die Untersuchungen von $\mathrm{H}$. Bächler nicht für den Beweis aus, daß die Knochenlager im Drachenloch und Wildenmannlisloch ihre Ursache in der Jagd des paläolithischen Menschen hätten. Aber wie jede exakte Analyse hat sie ihren Wert einmal darin, daß sie neue Unterlagen schafft, und zum andern, weil sie Anregung zu neuen Analysen gibt:

Wie die Untersuchung der Sedimente einer größeren Zahl von Höhlenbären-Höhlen gezeigt hat (E. SCHMID 1957, 1958), sind die meisten Höhlenbärenschichten ganz oder in einem Abschnitt während des Verlaufs der langen Vorstoßphase der Würm-Eiszeit abgelagert worden, wobei sich stellenweise die starken Schwankungen mit dem Frühwürm- 
Vorstoß und dem folgenden kräftigen Interstadial bis zum Haupt.Würm-Vorstoß nachweisen lassen. Wenn es zutrifft, daß das vorherrschende Sterben von Jungtieren auf mangelnde Ernährung zurückzuführen ist, dann müßte sich dies im verschieden starken Anteil von Jungtieren innerhalb der Schichtenfolge ausprägen.

\section{VII.}

Zur Beantwortung der Frage "Jagdbeute oder natürlicher Tod?" sollte - auch an anderen Fundstellen und bei anderen Tieren - nicht nur die osteologische Erfassung der Knochen und Zähne eine Rolle spielen. Was die Jagd anbetrifft, so können wir über die Altersstaffelung der Beute des paläolithischen Menschen vorerst nur unzureichend Auskunft geben, weil erst sehr wenige Stationen in dieser Hinsicht untersucht sind. Andererseits können von einer bekannten Fundstelle einer bestimmten Kultur aus nicht für andere Stellen gleicher Kultur, erst recht nicht anderer Kultur, über derartige Einzelheiten sichere Aussagen gemacht werden; denn der Mensch neigt auch in früher Zeit bei aller Traditionsgebundenheit doch auch dazu, durch veränderte äußere Verhältnisse, die wir vielleicht gar nicht erkennen können, oder gar aus innerem Antrieb, Neues zu erfinden. Das gleiche Ziel kann durch den Menschen auf die verschiedensten Weisen erreicht werden.

Vor allem aber wissen wir viel zu wenig über die Biologie der fossilen Säugetiere. Bei der paläobiologischen und prähistorischen Deutung von Knochenlagerstätten kann man die Ergebnisse der modernen Zoologie nicht außer Acht lassen: Physiologie, OKkologie, Genetik und Verhaltensforschung haben in den letzten 30 Jahren für die Säugetiere vielerlei neue Einsichten gebracht, die auch für die Deutung der Fossilien genutzt werden sollten. Die scharfe Ausprägung artgebundener Eigenheiten mahnen zur Vorsicht, wenn man Verhalten oder Eigentümlichkeiten einer Art auf eine andere Art übertragen will. Im Grunde wissen wir nichts über das Verhalten, kaum etwas über die spezifische Lebensweise des Höhlenbären. Aus dem bisher ergrabenen Material läßt sich nicht viel mehr herausholen, als was bisher veröffentlicht worden ist. Aber bei künftigen Ausgrabungen ungestörter Höhlenbärenschichten sollten neben den geologischen und prähistorischen Gesichtspunkten die Fragen der Paläobiologie und des tierlichen Verhaltens auch beim Freilegen der Funde mit geprütt werden. Dann nur können wir einen Schritt weiter über das bisher Erkannte hinaus kommen. Wie sehr solch neues, von den komplexen Fragen getragenes Ausgraben notwendig ist, sollte der vorliegende Aufsatz zeigen.

\section{$\mathrm{Sch}$ if ten-Nachweis}

Bächler, E.: Das Alpine Paläolithikum der Schweiz. - Monogr. z. Ur- u. Frühgesch. d. Schweiz, 2, Basel 1940.

BäCHLER, H.: Die Altersgliederung der Höhlenbärenreste im Wildkirchli, Wildenmannlisloch und Drachenloch. - Quartär 9, Bonn 1957.

Burckhardt, D.: Ưber das Wintersterben der Hirsche in der Umgebung des Nationalparkes. Z. Schweiz. Bund. f. Naturschutz 23, Basel 1957.

Marinelli, W.: Der Schädel des Höhlenbären. - In: Abel \& Kyrle, Die Drachenhöhle bei Mixnitz, Wien 1931.

Schмid, Elisabeth: Von den Sedimenten der Salzofenhöhle. - Sber. österr. Akad. Wiss., math.-nat. Kl., Abt. I, 166, S. 43-45, Wien 1957. - - Höhlenforschung und Sedimentanalyse. Schr. Inst. Ur- u. Frühgeschichte d. Schweiz, Nr. 13, Basel 1958.

Soergel, W.: Die Jagd der Vorzeit, - Jena 1922.

Manuskr. eingeg. 24. 6. 1959.

Anschrift d. Verf.: Prof. Dr. Elisabeth Schmid, Freiburg i. Br., Deichelweiherweg 17. 